\title{
NMR metabolomics of fibroblasts with inherited mitochondrial Complex I mutation reveals treatment-reversible lipid and amino acid metabolism alterations
}

\author{
Daniel Morvan ${ }^{1,2,4}$ (D) Aicha Demidem ${ }^{3}$
}

Received: 23 October 2017 / Accepted: 27 February 2018 / Published online: 22 March 2018

(c) The Author(s) 2018

\begin{abstract}
Introduction Elucidating molecular alterations due to mitochondrial Complex I (CI) mutations may help to understand CI deficiency (CID), not only in mitochondriopathies but also as it is caused by drugs or associated to many diseases.

Objectives CID metabolic expression was investigated in Leber's hereditary optic neuropathy (LHON) caused by an inherited mutation of CI.

Methods NMR-based metabolomics analysis was performed in intact skin fibroblasts from LHON patients. It used several datasets: one-dimensional ${ }^{1} \mathrm{H}-\mathrm{NMR}$ spectra, two-dimensional ${ }^{1} \mathrm{H}-\mathrm{NMR}$ spectra and quantified metabolites. Spectra were analysed using orthogonal partial least squares-discriminant analysis (OPLS-DA), and quantified metabolites using univariate statistics. The response to idebenone (IDE) and resveratrol (RSV), two agents improving CI activity and mitochondrial functions was evaluated.

Results LHON fibroblasts had decreased CI activity $(-43 \%, \mathrm{p}<0.01)$. Metabolomics revealed prominent alterations in LHON including the increase of fatty acids (FA), polyunsaturated FA and phosphatidylcholine with a variable importance in the prediction (VIP) $>1$ in OPLS-DA, $p<0.01$ in univariate statistics, and the decrease of amino acids (AA), predominantly glycine, glutamate, glutamine (VIP $>1$ ) and alanine (VIP $>1, \mathrm{p}<0.05$ ). In LHON, treatment with IDE and RSV increased CI activity ( +40 and $+44 \%, \mathrm{p}<0.05)$. IDE decreased FA, polyunsaturated FA and phosphatidylcholine $(\mathrm{p}<0.05)$, but did not modified AA levels. RSV decreased polyunsaturated FA, and increased several AA (VIP $>1$ and/or $\mathrm{p}<0.05$ ).

Conclusion LHON fibroblasts display lipid and amino acid metabolism alterations that are reversed by mitochondria-targeted treatments, and can be related to adaptive changes. Findings bring insights into molecular changes induced by CI mutation and, beyond, CID of other origins.
\end{abstract}

Keywords Mitochondrial Complex I deficiency $\cdot$ Leber's hereditary optic neuropathy $\cdot$ NMR-based metabolomics $\cdot$ Lipid and amino acid metabolism $\cdot$ Idebenone $\cdot$ Resveratrol

Electronic supplementary material The online version of this article (https://doi.org/10.1007/s11306-018-1345-9) contains supplementary material, which is available to authorized users.

Daniel Morvan

Daniel.Morvan@uca.fr

1 UCA University, boulevard François Mitterrand, 63001 Clermont-Ferrand, France

2 Comprehensive Cancer Centre Jean Perrin, rue Montalembert, 63011 Clermont-Ferrand, France

3 Faculty of Pharmacy, UMR1019 INRA/UCA, ECREIN, place Henri Dunant, 63001 Clermont-Ferrand, France

4 Department of Biophysics, Faculty of Medicine, Place Henri Dunant, 63001 Clermont-Ferrand, France

$\begin{array}{ll}\text { Abbreviations } \\ \text { CI } & \text { Mitochondrial Complex I } \\ \text { CID } & \text { Mitochondrial Complex I deficiency } \\ \text { LHON } & \text { Leber's hereditary optic neuropathy } \\ \text { ETC } & \text { Electron transport chain } \\ \text { EAAT1 } & \text { Excitatory amino acid transporter-1 } \\ \text { IDE } & \text { Idebenone } \\ \text { RSV } & \text { Resveratrol } \\ \text { HRMAS } & \text { High resolution magic angle spinning } \\ \text { OPLS-DA } & \text { Orthogonal partial least squares-discriminant } \\ & \text { analysis } \\ \text { VIP } & \text { Variable importance in the prediction } \\ \text { FA } & \text { Fatty acid } \\ \text { AA } & \text { Amino acid }\end{array}$


ER Endoplasmic reticulum

LD Lipid droplet

\section{Introduction}

Mitochondrial Complex I (NADH: ubiquinone oxidoreductase, CI) is a multimeric enzyme of the mitochondrial electron transport chain (ETC). CI subunit mutations, carried by either the nuclear or the mitochondrial genome, impair CI activity and are responsible for the most frequent mitochondrial diseases. Elucidating biochemical alterations in these diseases may help to understand CI deficiency (CID), not only in mitochondrial diseases, but also as it is caused by drugs targeting CI, or is a pathogenic component of diseases including neurodegeneration and cancer (Mimaki et al. 2012). To this aim, we investigated using metabolomics Leber's hereditary optic neuropathy (LHON), a mitochondrial disease caused by an inherited mutation of a mitochondrial DNA-encoded subunit of CI. LHON is a systemic disease although its main clinical expression is blindness associated to the loss of retinal ganglion cells (RGC) through a mechanism incompletely elucidated.

Metabolic abnormalities reported in LHON are few and include decreased CI activity, impairment of CI-driven respiration, increased ROS production, and decreased expression of excitatory amino acid transporter-1 (EAAT1) (Beretta et al. 2004). However, cells with CID have been shown to develop mechanisms of adaptation, including increased mitogenesis that may yield incomplete penetrance of the disease in LHON and AMP-activated protein kinase (AMPK) activation that improves cellular bioenergetics (Distelmaier et al. 2015).

Metabolomics can be expected to improve knowledge about molecular changes associated to CID. However, metabolomics studies of CID are few. A NMR-based metabolomics study on pharmacological inhibition of CI showed the decrease of amino acids (AA) and perturbations of phospholipid metabolism (Baykal et al. 2008). Another NMR study of myotubes exposed to rotenone showed changes in AA levels and increase in lactate (Lac) production (Xu et al. 2011). Using mass spectrometry (MS)-based metabolomics, it was shown that $\mathrm{CI}$ mutation in worms caused the decrease of most AA and succinate (Morgan et al. 2015). CI mutation in mice caused the increase of hydroxyacylcarnitines, in favor of decreased betaoxidation (Leong et al. 2012). A MS investigation in LHON fibroblasts reported the decrease of proteinogenic AA and changes in phospholipids (Chao de la Barca et al. 2016). A MS study of cells exposed to pharmacological inhibition of CI showed the decrease of glutamate (Glu), glutamine (Gln) and TCA cycle derivatives, and the increase of Lac (Janzer et al. 2014).
We here report an untargeted NMR-based metabolomics analysis of intact skin fibroblasts from LHON patients. LHON metabolomics is challenged by the low availability of samples and biological variability associated to the disease. We thus used orthogonally-filtered partial least squares discriminant analysis (OPLS-DA) in an attempt to reveal metabolic changes specifically related to LHON. In this study, OPLS-DA was applied to high resolution one-dimensional (1D) ${ }^{1} \mathrm{H}-\mathrm{NMR}$ spectra and two-dimensional (2D) ${ }^{1} \mathrm{H}-\mathrm{NMR}$ spectra whenever available. In addition, a set of metabolites was quantified from $1 \mathrm{D}^{1} \mathrm{H}-\mathrm{NMR}$ spectra.

Treatment of LHON remains a challenge. At present mitochondria-targeted agents remain the most beneficial. One of the most employed, idebenone (IDE), favors bypassing of CI directly to Complex III of the ETC (Angebault et al. 2011). Another beneficial agent, resveratrol (RSV), improves antioxidant defences in CI mutations (Mathieu et al. 2016) and protects against rotenone-induced apoptosis and neurodegeneration (Zhang et al. 2015). We thus sought for evaluating metabolic changes induced in LHON fibroblasts by IDE and RSV.

In this article, it is shown that LHON fibroblasts exhibit two prominent alterations, the increase of lipids and the decrease of AA, which are reversed by treatments. These data shed light on metabolic and molecular alterations in CID.

\section{Materials and methods}

\subsection{Patients}

Patient demographic and genetic data are given in Table S1. The study was approved by the Ethics Committee of the University Hospital of Angers (No. 2011/39). All patients gave informed consent. A skin biopsy was obtained from 5 affected LHON patients and 4 healthy subjects. Healthy subjects were males, aged between 31 and 59, without any personal or familial history of neurological or genetic disease.

\subsection{Cell culture and treatment}

Fibroblasts were cultured according to a published protocol (Loiseau et al. 2007). Ethanol, used as the vehicle, idebenone (IDE, Santhera Pharmaceutical, Liestal, Switzerland) at $10 \mu \mathrm{M}$, and resveratrol (RSV, Sigma Chemicals, St. Louis, MO) at $50 \mu \mathrm{M}$ were added $24 \mathrm{~h}$ before cell collection. Doses of IDE and RSV were selected from literature data (Angebault et al. 2011; Mathieu et al. 2016).

For NMR spectroscopy analysis, $5-10 \times 10^{6}$ cells were washed twice in $500 \mu \mathrm{L} \mathrm{D} \mathrm{D}_{2} \mathrm{O}$, then frozen in liquid nitrogen and kept at $-20{ }^{\circ} \mathrm{C}$ until analysis. Primary cell cultures were performed twice with vehicle-exposed NL fibroblasts $(n=8$ 
samples from 4 healthy subjects) and LHON patient fibroblasts ( $\mathrm{n}=10$ from 5 patients). Other LHON fibroblast cultures were treated with IDE (L-IDE, $\mathrm{n}=8$ ) or RSV (L-RSV, $\mathrm{n}=9$ ) and compared to NL fibroblast cultures treated with IDE (N-IDE, $n=7)$ or RSV (N-RSV, $n=6)$.

\subsection{Complex I activity measurement}

Complex I (CI, NADPH ubiquinone reductase, EC 1.6.5.3) activity was measured according to a procedure described elsewhere (Loiseau et al. 2007). Citrate synthase (CS, EC 2.3.3.1) activity was assayed by a standard procedure, and used to correct $\mathrm{CI}$ activity for mitochondrial mass in the CIto-CS activity ratio.

\subsection{NMR spectroscopy}

NMR spectroscopy was performed on a small-bore 500$\mathrm{MHz}$ Bruker Avance DRX spectrometer equipped with a high resolution magic angle spinning (HRMAS) probe enabling to analyze intact cells. Freshly unfrozen cell pellets were set into $4 \mathrm{~mm}$-diameter, $50-\mu \mathrm{L}$ zirconium oxide rotor tubes. The rotors were spun at $4 \mathrm{kHz}$ and maintained at $4{ }^{\circ} \mathrm{C}$, using the BCU-05 temperature unit. One-dimensional ${ }^{1} \mathrm{H}-\mathrm{NMR}$ spectra were obtained using a nuclear Overhauser enhancement spectroscopy sequence with lowpower water-signal presaturation (NOESYPR) during both the 3.8-s relaxation delay and the 100-ms mixing time of the sequence. The spectral width was $12 \mathrm{ppm}$ with 16,384 complex data points and 32 transients.

A subset of intact cell pellets kept at $4{ }^{\circ} \mathrm{C}$ underwent a 2D ${ }^{1} \mathrm{H}-\mathrm{NMR}$ Total Correlation Spectroscopy (TOCSY) sequence with water signal suppression at low power, 6-ppm spectral bandwidth along both axes, $256 \times 2048$ matrix, 75-ms mixing time, during which was applied the spin-lock pulse train (DIPSI-2), 1-s relaxation delay, and 16 repetitions.

\subsection{Data processing}

One dimensional NMR spectra were transferred to the MestReNova software (Mestrelab Research, Santiago de Compostela, Spain). A standardized phase correction was applied. The residual water signal was suppressed between 4.5 and $5.3 \mathrm{ppm}$. Strongly contributing signals of HEPES (between 3.08 and 3.19 ppm, and between 3.85 and $3.92 \mathrm{ppm})$ and ethanol (1.18 and $3.62 \mathrm{ppm})$ were removed. Then spectra at full resolution were normalized to the total spectral area, and transferred to the SIMCA 14 software (Umetrics, Umea, Sweden) for data processing.

TOCSY spectra were reconstructed at $256 \times 256$ then transferred to the Matlab 7 software (Mathworks, Natik, MA). The residual water signal was removed from spectra along both spectral axes. TOCSY spectra were normalized to the total spectral area, then linearized and transferred to the SIMCA 14 software for data processing. OPLS-DA results were reconstructed into 2D matrices along spectral coordinates using Matlab 7.

As a third set of NMR data, metabolites giving rise to signals without superposition (or with easy to handle superposition), or with low intensity but sufficient signal-to-noise ratio in 1D NMR spectra were quantified $(n=21)$. The attribution of signals was based on literature data. Signals were integrated using MestReNova. Metabolite relative concentrations were calculated by normalizing the metabolite signal to the residual Lys protein signal at $2.99 \mathrm{ppm}$, by adaptation of a technique developed for $2 \mathrm{D}{ }^{1} \mathrm{H}$ NMR HRMAS quantification (Bayet-Robert et al. 2010).

\subsection{Statistical data analysis}

Data was scaled using the unit variance method, then processed by Orthogonal Partial Least Squares Discriminant Analysis (OPLS-DA) using the SIMCA 14 software (Umetrics, Uppsala, Sweden).

First OPLS-DA removes data that is not correlated to classes. Remaining data is fitted to a linear combination of variables. The obtained component or predictive component is tested for significance using a permutation algorithm (Monte-Carlo cross validation algorithm) by the SIMCA 14 software. If it is significant, other components, orthogonal to the first component and between one another, are generated as long as they are statistically significant, based on the built-in cross-validation test of the SIMCA 14 software.

The overall quality of the model is described by R2X, $\mathrm{R} 2 \mathrm{Y}$ and $\mathrm{Q} 2$ parameters that designate the explained fraction of variance of data (R2X), the explained fraction of variance of classes (R2Y), and the cross-validated fraction of variance of classes $(\mathrm{Q} 2)$, respectively.

For each analysis, OPLS-DA provided:

(i) A scores plot (t[1] vs. t[0]) displaying individuals projected on the first two components.

(ii) A loadings plot ( $\mathrm{p}[1])$, displaying variables projected on the first component.

(iii) A correlation table ( $\mathrm{p}(\mathrm{corr})[1])$, giving the correlation coefficient of scores with data for each variable. A plot of un-normalized $\mathrm{p}$ [1] loadings against the chemical shift, combined with $\mathrm{p}$ (corr)[1] was used to display $1 \mathrm{D}{ }^{1} \mathrm{H}-\mathrm{NMR}$ spectrum-derived OPLS-DA models. Variables that combine high strength $\mathrm{p}$ [1] and pcorr[1] contribute the most to the model.

(iv) A variable importance for the projection (VIP) table. VIP $>1$ was the criterion for statistical significance of a variable. 
Univariate statistical analysis used the unpaired Student's $t$ test. Differences were considered significant at $\mathrm{p}<0.05$.

\section{Results}

\subsection{LHON fibroblasts exhibit increased lipid and decreased amino acid levels}

The CI-to-CS activity ratio was decreased in LHON $(62 \pm 11$ vs. $109 \pm 7 \%$, LHON vs. NL, p <0.01) (Fig. S1).

With OPLS-DA, the use of a single 2-class covariable only provided statistically significant results. First, OPLSDA of $1 \mathrm{D}^{1} \mathrm{H}$ NMR spectra (LHON vs. NL, $\mathrm{n}=10$ vs. $\mathrm{n}=8$ ) yielded a good quality model $(\mathrm{R} 2 \mathrm{X}=0.60, \mathrm{R} 2 \mathrm{Y}=0.88$ and $\mathrm{Q} 2=0.58)$, with one predictive and 2 orthogonal components. The scores plot showed a clear separation between classes (Fig. 1a).

In the loading plot (Fig. 1b; Table 1), several NMR signals were increased (VIP $>1$ and pcorr[1] $>+0.40$ ) in LHON, including those of tFA, PUF and PtC.
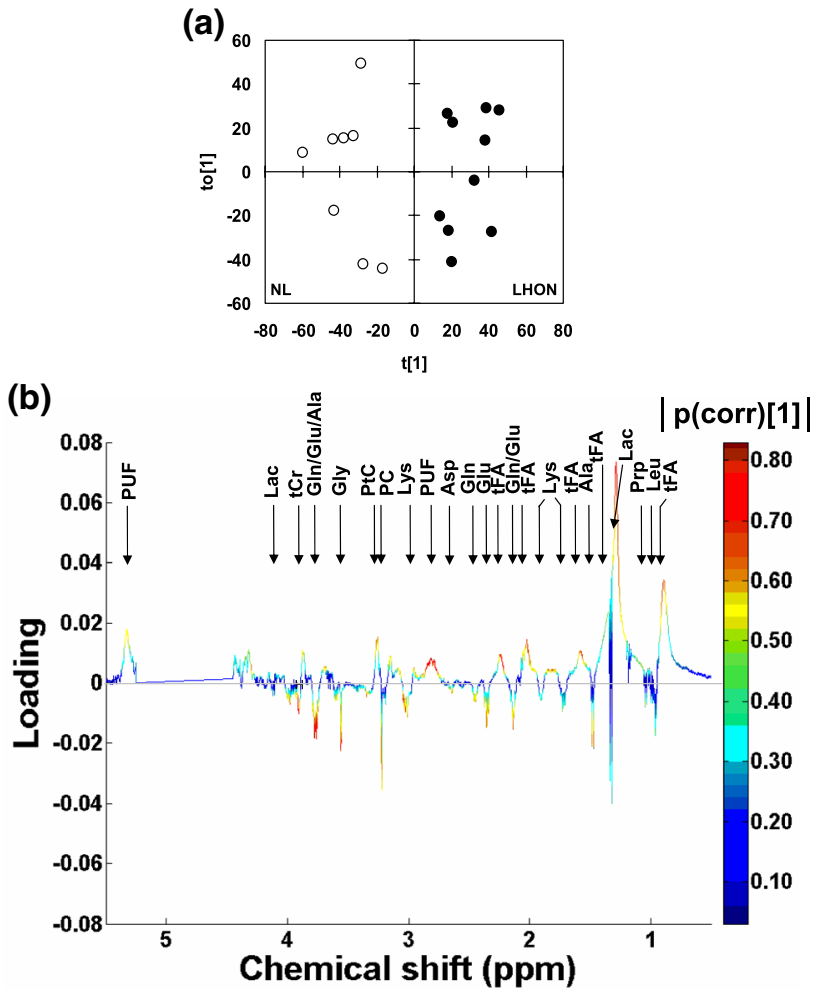

Fig. 1 a, b OPLS-DA of $1 \mathrm{D}{ }^{1} \mathrm{H}-\mathrm{NMR}$ spectra of LHON vs. NL. NL, $\mathrm{n}=8$, vs. $\mathrm{LHON}, \mathrm{n}=10 . \mathrm{R} 2 \mathrm{X}=0.60, \mathrm{R} 2 \mathrm{Y}=0.88$ and $\mathrm{Q} 2=0.58,1$ predictive and 2 orthogonal components. a Scores plot (t[1] vs. $\mathrm{t}[0]$ ). White dots, NL; black dots, LHON. b Loadings plot on the predictive component. Main assignment is given. Full assignment and metabolite abbreviations, see Table 1; positive loading, increased in LHON; negative loading, decreased in LHON; color-coding, strength of the correlation coefficient
Several NMR signals were decreased in LHON including those of several AAs: Gly, Ala, Glu, Gln, Asp, Lys, and collectively Glu/GIn/Ala/Lys, Glu/Gln and Leu/Ileu/Val. Other signals were decreased including those of $\mathrm{tCr}, \mathrm{PC}$, PE and Prp.

Second, we applied OPLS-DA to 2D TOCSY spectra (LHON vs. NL, $n=6$ vs. $n=6$ ). The obtained model had good quality $(\mathrm{R} 2 \mathrm{X}=0.85, \mathrm{R} 2 \mathrm{Y}=1$ and $\mathrm{Q} 2=0.42)$, with one predictive and 7 orthogonal components. The scores plot showed a clear separation between classes (Fig. 2a). In the loading plot (Fig. 2b; Table 2), some off-diagonal signals were significantly increased in LHON including those of tFA and PUF. Most significantly decreased off-diagonal signals in LHON included those of several amino acids: Val, Arg, Ala, Lys, Asp, Ser and Phe.

Then, we applied univariate analysis and OPLS-DA to the set of quantified metabolites. Using univariate statistics (Table 3), lipid derivatives (tFA, PUF and PtC) were increased in LHON $(+50$ to $+117 \%$, LHON vs. NL, all $\mathrm{p}<0.05)$. AA were decreased in LHON, including Phe, Tyr, Gly and Ala ( -24 to $-40 \%$, LHON vs. NL, $\mathrm{p}<0.05)$. Also Prp was decreased $(-41 \%$, LHON vs. NL, $\mathrm{p}<0.05)$. OPLSDA of quantified metabolites yielded a good quality model $(\mathrm{RX} 2=0.59, \mathrm{RY} 2=0.91$ and $\mathrm{Q} 2=0.75)$ with 1 predictive and 2 orthogonal components. Significantly increased metabolites in LHON were PUF, PtC, tFA and MyI, and significantly decreased metabolites were Gln, Ala, Gly, Phe, Tyr and Prp (Fig. S2a,c, Table S2).

\subsection{IDE reverses lipid changes in LHON fibroblasts}

The CI-to-CS activity ratio increased in $\mathrm{LHON}$ in response to IDE $(87 \pm 18$ vs. $62 \pm 11 \%$, L-IDE vs. LHON, p <0.05) (Fig. S1).

The OPLS-DA of 1D NMR spectra failed to provide statistically significant models separating N-IDE and NL or L-IDE and LHON.

The quantification of metabolites in response to IDE is given in Table 3. There were no significant changes in $\mathrm{N}$-IDE vs. NL. In contrast, in LHON, IDE decreased tFA, PUF and PtC (L-IDE vs. LHON, $n=8$ vs. $n=10, p<0.05$ ). The OPLS-DA of quantified metabolites yielded no significant model separating N-IDE and NL or L-IDE and LHON.

\subsection{RSV partly reverses lipid and amino acid changes in LHON fibroblasts}

The CI-to-CS activity ratio increased in $\mathrm{LHON}$ in response to $\mathrm{RSV}(89 \pm 25$ vs. $62 \pm 11 \%$, L-RSV vs. LHON, p <0.05) (Fig. S1).

The OPLS-DA of 1D NMR spectra did not provide statistically significant models separating N-RSV and NL. 
Table 1 OPLS-DA of $1 \mathrm{D}^{1} \mathrm{H}-\mathrm{NMR}$ spectra of LHON vs. NL and L-RSV vs. LHON

\begin{tabular}{|c|c|c|c|c|}
\hline \multirow[t]{2}{*}{ Chemical shift (ppm) } & \multicolumn{2}{|c|}{ Metabolite assignment } & \multicolumn{2}{|l|}{ VIP (pcorr[1]) } \\
\hline & Abbreviation & Full name & LHON vs. NL & L-RSV vs. LHON \\
\hline 5.33 & PUF & Polyunsaturated FA $(\mathrm{CH}=\mathrm{CH})$ & $1.28(+0.51)$ & $1.45(-0.40)$ \\
\hline 4.20 & $\mathrm{PC}$ & Phosphocholine $\left(\mathrm{CH}_{2}-\mathrm{O}\right)$ & - & $1.89(+0.55)$ \\
\hline 4.12 & Lac & Lactate $(\mathrm{CH})$ & - & $1.76(+0.50)$ \\
\hline 4.06 & MyI & Myoinositol (2-CH) & - & $1.26(+0.33)$ \\
\hline 3.99 & PE & Phosphoethanolamine $\left(\mathrm{CH}_{2}-\mathrm{O}\right)$ & $1.16(-0.52)$ & $1.50(+0.41)$ \\
\hline 3.92 & $\mathrm{tCr}$ & Total creatine $\left(\mathrm{CH}_{2}\right)$ & $1.67(-0.80)$ & $1.57(+0.44)$ \\
\hline 3.77 & Glu/Gln/Ala & Glutamate/glutamine/alanine $(\mathrm{CH})$ & $1.79(-0.86)$ & $2.07(+0.61)$ \\
\hline 3.62 & $\mathrm{PC}$ & Phosphocholine $\left(\mathrm{CH}_{2}-\mathrm{N}\right)$ & $1.34(-0.59)$ & $1.72(+0.49)$ \\
\hline 3.56 & Gly & Glycine $\left(\mathrm{CH}_{2}\right)$ & $1.61(-0.76)$ & $2.08(+0.61)$ \\
\hline 3.53 & MyI & Myoinositol (1,3-CH) & - & $2.44(+0.73)$ \\
\hline 3.43 & Tau & Taurine $\left(\mathrm{N}-\mathrm{CH}_{2}\right)$ & - & $1.29(-0.34)$ \\
\hline 3.26 & $\mathrm{PtC}$ & Phosphatidylcholine $\left(\left(\mathrm{CH}_{3}\right)_{3}-\mathrm{N}\right)$ & $1.36(+0.66)$ & $1.12(+0.29)$ \\
\hline 3.22 & $\mathrm{PC}$ & Phosphocholine $\left(\left(\mathrm{CH}_{3}\right)_{3}-\mathrm{N}\right)$ & $1.45(-0.66)$ & $1.86(+0.54)$ \\
\hline 3.20 & Cho & Choline $\left(\left(\mathrm{CH}_{3}\right)_{3}-\mathrm{N}\right)$ & - & $1.30(-0.34)$ \\
\hline 3.03 & $\mathrm{tCr}$ & Total creatine $\left(\mathrm{CH}_{3}\right)$ & $1.60(-0.76)$ & $1.07(+0.25)$ \\
\hline 2.82 & PUF & Polyunsaturated $\mathrm{FA}\left(=\mathrm{CH}-\mathrm{CH}^{2}-\mathrm{CH}=\right)$ & $1.76(+0.85)$ & $1.91(-0.56)$ \\
\hline 2.64 & Asp & Aspartate $\left(\beta-\mathrm{CH}_{2}\right)$ & $1.34(-0.64)$ & $1.35(+0.36)$ \\
\hline 2.55 & GSx & Total glutathione $\left(\gamma-\mathrm{CH}_{2}\right)$ & - & $1.23(-0.35)$ \\
\hline 2.44 & Gln & Glutamine $\left(\gamma-\mathrm{CH}_{2}\right)$ & $1.44(-0.63)$ & $1.32(+0.35)$ \\
\hline 2.35 & Glu & Glutamate $\left(\gamma-\mathrm{CH}_{2}\right)$ & $1.45(-0.67)$ & $1.17(+0.35)$ \\
\hline 2.25 & $\mathrm{tFA}$ & Total FA $\left(\mathrm{CH}_{2}-\mathrm{CO}\right)$ & $1.72(+0.81)$ & $1.72(-0.49)$ \\
\hline 2.12 & Glu/Gln/GSx & Glutamate/glutamine/glutathione $\left(\beta-\mathrm{CH}_{2}\right)$ & $1.55(-0.70)$ & $1.47(+0.40)$ \\
\hline 2.03 & $\mathrm{tFA}$ & Total FA $\left(\mathrm{CH}_{2}-\mathrm{CH}=\right)$ & $1.56(+0.72)$ & $1.29(-0.33)$ \\
\hline 1.90 & Lys & Lysine $\left(\beta-\mathrm{CH}_{2}\right)$ & $1.05(-0.42)$ & $1.28(+0.33)$ \\
\hline 1.72 & & Lysine $\left(\delta-\mathrm{CH}_{2}\right)$ & $1.15(-0.41)$ & - \\
\hline 1.59 & tFA & Total FA $\left(\mathrm{CH}_{2}-\mathrm{CH}_{2}-\mathrm{CO}\right)$ & $1.57(+0.72)$ & $1.08(-0.24)$ \\
\hline 1.47 & Ala & Alanine $\left(\mathrm{CH}_{3}\right)$ & $1.46(-0.70)$ & $1.81(+0.52)$ \\
\hline 1.33 & Lac & Lactate $\left(\mathrm{CH}_{3}\right)$ & - & $1.76(+0.50)$ \\
\hline 1.29 & $\mathrm{tFA}$ & total FA $\left(-\left(\mathrm{CH}_{2}\right)_{\mathrm{n}}-\right)$ & $1.58(+0.75)$ & $1.03(-0.24)$ \\
\hline 1.05 & Prp & Propionate $\left(\mathrm{CH}_{3}\right)$ & $1.10(-0.49)$ & $1.80(+0.52)$ \\
\hline $0.96-1.02$ & Leu/Ileu/Val & Leucine/isoleucine/valine $\left(\mathrm{CH}_{3}\right)$ & $1.04(-0.44)$ & $1.58(+0.44)$ \\
\hline 0.90 & $\mathrm{tFA}$ & Total FA $\left(\mathrm{CH}_{3}\right)$ & $1.59(+0.73)$ & $1.10(-0.26)$ \\
\hline
\end{tabular}

The first column gives chemical shift of $1 \mathrm{D}^{1} \mathrm{H}-\mathrm{NMR}$ signals. The second and third columns give signal assignment with abbreviation and full name of the metabolite(s). The last 2 columns give VIP when $>1$ (statistically significant variation), with p(corr)[1] in parentheses

Dash, not significant. The sign of $\mathrm{p}$ (corr)[1] indicates the direction of variation: negative, decrease; positive, increase

The OPLS-DA of $1 \mathrm{D}{ }^{1} \mathrm{H}-\mathrm{NMR}$ spectra (L-RSV vs. LHON, $n=9$ vs. $n=10$ ) yielded a good quality model $(\mathrm{R} 2 \mathrm{X}=0.96, \mathrm{R} 2 \mathrm{Y}=1$ and $\mathrm{Q} 2=0.54)$, with one predictive and 7 orthogonal components. The scores plot showed a clear separation between classes (Fig. 3a).

In the loading plot (Fig. 3b; Table 1) several NMR signals were decreased under RSV treatment including those of tFA at some spectral positions and PUF. Other NMR signals were increased including those of Glu/Gln, Gly, Ala and branched-chain AA. Three metabolites were specifically increased under RSV: PC, Lac and MyI.
The quantification of metabolites in response to RSV is given in Table 3. In L-RSV vs. LHON, Phe, MyI, PC (all, $\mathrm{p}<0.05)$ and $\mathrm{Lac}(\mathrm{p}<0.01)$ were increased and Tau was decreased $(\mathrm{p}<0.01)$. OPLS-DA of quantified metabolites was not significant in N-RSV vs. NL. In L-RSV vs. LHON, OPLS-DA yielded a good quality model $(\mathrm{RX} 2=0.48$, $\mathrm{RY} 2=0.98$ and $\mathrm{Q} 2=0.77$ ) with one predictive and 2 orthogonal components. Significantly increased metabolites in L-RSV were ATP, MyI, PC, Lac, Ala and Glu, and significantly decreased metabolites were PUF and Tau (Fig. S2b, d, Table S2). 
(a)
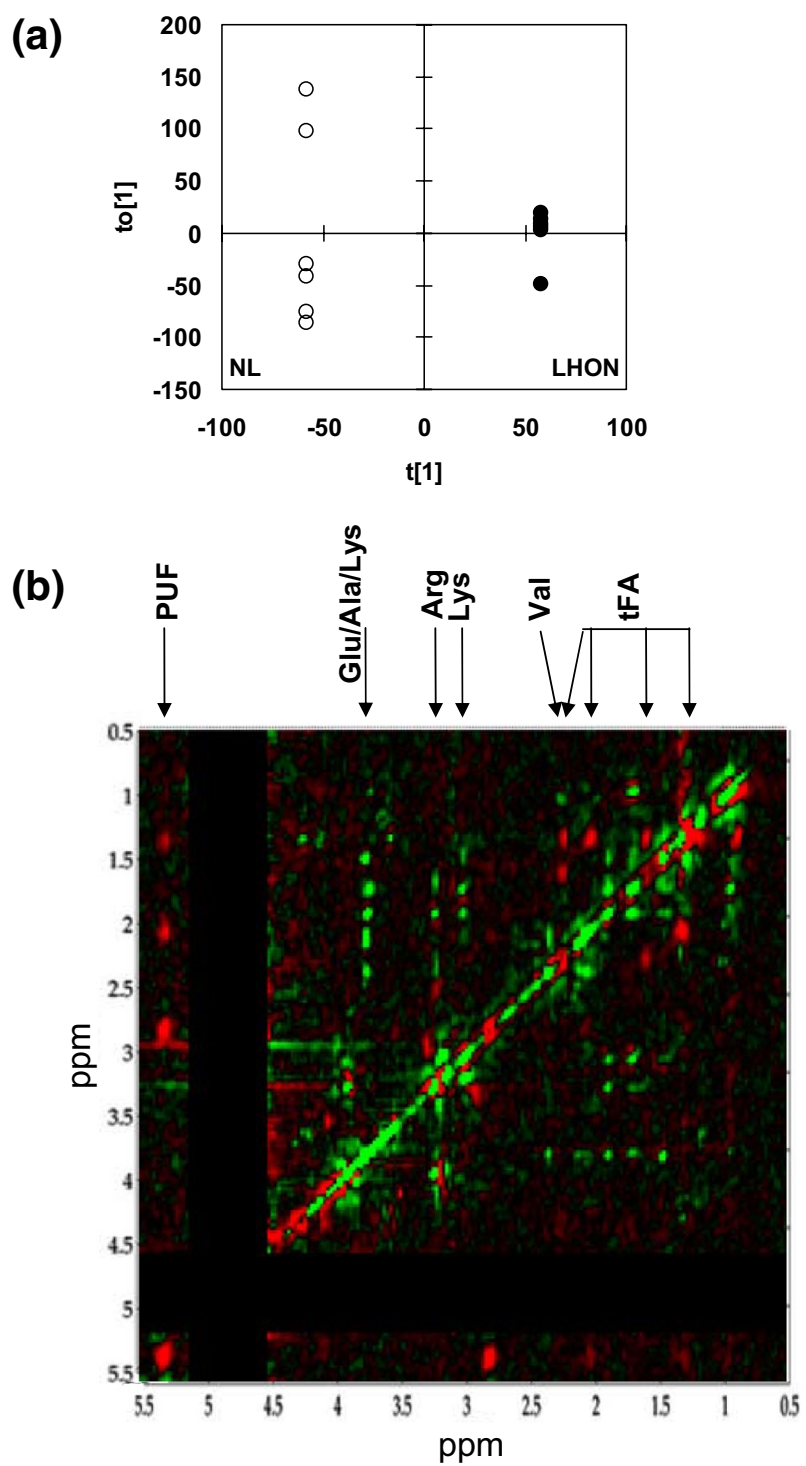

Fig. 2 a, b OPLS-DA of 2D TOCSY spectra of LHON vs. NL. NL, $\mathrm{n}=6$, vs. $\mathrm{LHON}, \mathrm{n}=6 . \mathrm{R} 2 \mathrm{X}=0.85, \mathrm{R} 2 \mathrm{Y}=1$ and $\mathrm{Q} 2=0.42,1$ predictive and 7 orthogonal components. a Scores plot (t[1] vs. $t[0])$. White dots, NL; black dots, LHON. b Loadings plot on the predictive component. It is color-coded according to the sign and strength of loadings. Main assignment of loadings is given. Full assignment and metabolite abbreviations, see Table 2; red loading, increased in LHON; green loading, decreased in LHON; biphasic, HEPES which chemical shifts are sensitive to physicochemical conditions

\section{Discussion}

This article demonstrates major metabolic alterations in LHON fibroblasts including the increase of lipids and the decrease of AA. These alterations were corrected by mitochondria-targeted treatments that, at least partly, alleviate CID. Our data provide insights into metabolic and molecular adaptive changes to CID.

\subsection{Increased lipid levels in CID}

We found increased fatty acid (FA) signals in LHON. Several studies have shown that NMR-visible FA signals originated from FA in triglycerides and cholesterol esters of lipid droplets (LD) (Boren and Brindle 2012). LD are synthesized in the endoplasmic reticulum (ER), and play a major role to store and transport FA, prevent potential cytotoxicity of free FA, and participate to the communication between the ER and mitochondria. Actually, the interaction between mitochondria and ER is very tight, both these organelles being implicated in not only lipid metabolism, but also $\mathrm{Ca}^{2+}$ signaling, bioenergetics, inflammation, autophagy and apoptosis.

Alteration in lipid metabolism has been shown in CID. Rotenone, a CI inhibitor, alters FA biosynthesis and betaoxidation in neuroblastoma cells (Worth et al. 2014) or induces triacylglycerol deposition in muscle-derived cells (He et al. 2013). Also, inhibition of beta-oxidation was reported in CI mutation (Leong et al. 2012).

In LHON, we also found changes in phospholipid derivatives and Prp. PtC was increased and PC decreased, suggesting increased $\mathrm{PtC}$ biosynthesis, a condition for LD formation. Prp in the form of propionyl-CoA, is synthesized by incomplete beta-oxidation of odd-chain FA in peroxisomes. Since beta-oxidation in peroxisomes requires reoxidation of NADH by mitochondria, the decrease of Prp could result from the inhibition of beta-oxidation in peroxisomes. Alternatively, Prp is a by-product of the degradation of branchedchain AA that, as discussed below, were decreased in LHON.

Overall, the increase of intracellular levels of lipids, especially FA, in LHON can be related to the reduction of mitochondrial/peroxisomal lipid beta-oxidation and the increase of LD formation.

\subsection{Decreased amino acid levels in CID}

We found decreased intracellular levels of free AA in LHON, in agreement with several reports in cells with deficient ETC. A NMR metabolomics analysis of an inherited neurodegeneration reported the decrease of Gly and Glurelated AA (Graham et al. 2016). A MS-metabolomics investigation of LHON fibroblasts showed the decrease of proteinogenic AA (Chao de la Barca et al. 2016). A study on pharmacological inhibition of ETC complexes reported the decrease of several AA (Baykal et al. 2008). A MS study of the response of transformed cells to $\mathrm{CI}$ inhibitors reported the decrease of Glu and Gln (Janzer et al. 2014).

Intracellular levels of free AA result from the balance between uptake, protein synthesis, oxidation and recycling by autophagy. AA oxidation through the TCA cycle should not be activated in LHON. As well, increased protein synthesis due to mitogenesis should not explain the magnitude of AA variations. 
Table 2 OPLS-DA of 2D TOCSY spectra of LHON vs. NL

\begin{tabular}{|c|c|c|c|}
\hline \multirow[t]{2}{*}{ Chemical shift (ppm) } & \multicolumn{2}{|l|}{ Metabolite } & \multirow{2}{*}{$\begin{array}{l}\text { VIP (pcorr[1]) } \\
\text { LHON vs. NL }\end{array}$} \\
\hline & Abbreviation & Full name & \\
\hline $5.33 \times 2.82$ & PUF & Polyunsaturated FA & $1.49(+0.56)$ \\
\hline $5.33 \times 2.03$ & & & $1.69(+0.65)$ \\
\hline $5.33 \times 1.29$ & & & $1.60(+0.61)$ \\
\hline $4.20 \times 3.58$ & $\mathrm{PC}$ & Phosphocholine & $1.37(-0.49)$ \\
\hline $4.17 \times 2.03$ & Pro & Proline & $1.19(-0.43)$ \\
\hline $4.12 \times 1.33$ & Lac & Lactate & - \\
\hline $4.06 \times(3.53-3.61)$ & MyI & Myoinositol & - \\
\hline$(3.53-3.61) \times 3.27$ & & & - \\
\hline $3.99 \times 3.22$ & $\mathrm{PE}$ & Phosphoethanolamine & $1.19(-0.40)$ \\
\hline $3.99 \times 3.13$ & Phe & Phenylalanine & $1.39(-0.50)$ \\
\hline $3.99 \times(2.88-2.95)$ & Asn & Asparagine & $1.19(-0.49)$ \\
\hline $3.97 \times 3.83$ & Ser & Serine & $1.44(-0.55)$ \\
\hline $3.89 \times(2.65-2.75)$ & Asp & Aspartate & $1.36(-0.50)$ \\
\hline $3.58 \times 1.33$ & Thr & Threonine & $1.15(-0.41)$ \\
\hline $3.77 \times 2.35$ & Glu & Glutamate & $1.24(-0.48)$ \\
\hline $2.35 \times(2.04-2.11)$ & & & $1.10(-0.40)$ \\
\hline $3.77 \times 1.90$ & Lys & Lysine & $1.39(-0.51)$ \\
\hline $3.77 \times 1.71$ & & & $1.49(-0.56)$ \\
\hline $3.77 \times 1.46$ & Ala & Alanine & $1.47(-0.56)$ \\
\hline $3.43 \times 3.27$ & Tau & Taurine & - \\
\hline $3.22 \times(1.65-1.90)$ & Arg & Arginine & $1.59(-0.61)$ \\
\hline $2.55 \times 2.17$ & GSx & Total glutathione & $1.31(-0.47)$ \\
\hline $2.44 \times 2.14$ & Gln & Glutamine & $1.20(-0.41)$ \\
\hline $2.28 \times 1.00$ & Val & Valine & $1.63(-0.62)$ \\
\hline $1.71 \times 0.95$ & Leu & Leucine & $1.18(-0.40)$ \\
\hline $1.29 \times 2.25$ & $\mathrm{tFA}$ & Total FA & $1.25(+0.43)$ \\
\hline $1.29 \times 2.03$ & & & $1.29(+0.48)$ \\
\hline $1.29 \times 0.90$ & & & $1.33(+0.51)$ \\
\hline
\end{tabular}

The first column gives chemical shifts of 2D TOCSY off-diagonal signals. The second and third columns give assignment with abbreviation and full name of the metabolite. The last column gives VIP when $>1$ (statistically significant variation), with $\mathrm{p}$ (corr)[1] in parentheses

Dash, not significant. The sign of $\mathrm{p}(\mathrm{corr})[1]$ indicates the direction of variation: negative, decrease; positive, increase
Membrane AA transport often depends on the redox status of the cell and/or requires the availability of ATP. A reduction in the intracellular transport of Glu, through decreased activity of EAAT1, was reported in LHON cybrids (Beretta et al. 2004). In addition, the reduction in plasma membrane import of AA may be caused by AMPK activation, an adaptive mechanism in CID (Distelmaier et al. 2015).

Autophagy is another mechanism that regulates AA levels. Low levels of AA activate autophagy through the inhibition of the mTOR pathway. The accumulation of autophagosomes was reported in LHON, in favor of the activation of autophagy (Dombi et al. 2016). In addition, mitogenesis, another adaptive mechanism in CID, often combines with mitophagy in the control of mitochondrial quality. Completion of the autophagic process requires fusion of autophagosomes with lysosomes, a mechanism that, despite activation of autophagy, may be impaired in neurodegeneration.

Overall, the decrease of intracellular levels of AA in LHON fibroblasts can be related to decreased membrane uptake/cytosolic transport of AA, or activated autophagy.

\subsection{Reversion of metabolic changes with mitochondria-targeted treatments}

In LHON, IDE increased the activity of CI, in agreement with other reports (Angebault et al. 2011). IDE completely reversed FA changes. IDE is an analogue of ubiquinone and favors bypassing of CI directly to Complex III of the ETC. 
Table 3 Relative concentrations of $1 \mathrm{D}{ }^{1} \mathrm{H}-\mathrm{NMR}$ quantified metabolites

\begin{tabular}{|c|c|c|c|c|c|c|c|c|}
\hline \multicolumn{2}{|l|}{ Metabolite } & \multirow{2}{*}{$\begin{array}{l}\text { Chemical shift } \\
(\mathrm{ppm})\end{array}$} & \multicolumn{6}{|c|}{ Concentration relative to NL (NL to LHON) or LHON (L-IDE and L-RSV) } \\
\hline Abbreviation & Full name & & NL $(n=8)$ & N-IDE $(n=7)$ & N-RSV $(n=6)$ & $\operatorname{LHON}(n=10)$ & L-IDE $(n=8)$ & L-RSV $(n=9)$ \\
\hline ATP & $\begin{array}{l}\text { Adenosine } \\
\text { triphosphate }\end{array}$ & $\begin{array}{l}8.54 \mathrm{~s} ; 8.12 ; \\
\quad 6.15\end{array}$ & $1.00(1.08)$ & $1.23(0.80)$ & $1.94(1.56)$ & $0.94(0.51)$ & $1.25(0.40)$ & $1.66(1.14)$ \\
\hline For & Formate & $8.46 \mathrm{~s}$ & $1.00(0.81)$ & $0.90(1.13)$ & $0.74(0.39)$ & $1.16(1.39)$ & $0.56(0.36)$ & $0.44(0.26)$ \\
\hline AMP & $\begin{array}{l}\text { Adenosine } \\
\text { monophosphate }\end{array}$ & $\begin{array}{l}8.26 \mathrm{~s} ; 8.36 \\
\quad 6.10\end{array}$ & $1.00(0.50)$ & $2.66(3.60)$ & $0.64(0.35)$ & $3.71(5.41)$ & $0.12(0.07)$ & $0.25(0.27)$ \\
\hline Phe & Phenylalanine & $7.42 \mathrm{~m} ; 7.33$ & $1.00(0.33)$ & $0.98(0.28)$ & $1.03(0.40)$ & $0.76(0.26)^{*}$ & $1.12(0.27)$ & $1.32(0.40)^{\$}$ \\
\hline Tyr & Tyrosine & $7.16 \mathrm{~m} ; 6.90$ & $1.00(0.40)$ & $1.00(0.28)$ & $1.03(0.44)$ & $0.67(0.37)^{*}$ & $1.20(0.27)$ & $1.31(0.44)$ \\
\hline UXP & $\begin{array}{l}\text { Uridine phos- } \\
\text { phate deriva- } \\
\text { tives }\end{array}$ & $5.97 \mathrm{~m} ; 7.94$ & $1.00(0.67)$ & $1.00(0.39)$ & $1.11(0.69)$ & $1.14(0.48)$ & $1.09(0.29)$ & $1.37(0.73)$ \\
\hline MyI & Myoinositol & $\begin{array}{c}4.06 \mathrm{~m} ; 3.27 \\
3.53 ; 3.61\end{array}$ & $1.00(0.63)$ & $2.20(1.98)$ & $5.41(3.17)^{\$ \$}$ & $2.15(1.23)$ & $0.88(0.54)$ & $1.92(1.03)^{\$}$ \\
\hline $\mathrm{tCr}$ & Total creatine & $3.92 \mathrm{~s} ; 3.03$ & $1.00(1.28)$ & $0.72(0.27)$ & $0.92(0.70)$ & $0.81(0.28)$ & $1.22(0.49)$ & $0.85(0.48)$ \\
\hline Gly & Glycine & $3.56 \mathrm{~s}$ & $1.00(0.43)$ & $0.92(0.25)$ & $0.99(0.32)$ & $0.69(0.20)^{*}$ & $1.18(0.24)$ & $1.20(0.23)$ \\
\hline Tau & Taurine & $3.43 t ; 3.27$ & $1.00(0.41)$ & $1.12(0.44)$ & $1.28(0.52)$ & $1.47(0.68)$ & $0.63(0.17)^{\#}$ & $0.53(0.24)^{\$ \$}$ \\
\hline $\mathrm{PtC}$ & $\begin{array}{l}\text { Phosphatidylcho- } \\
\text { line }\end{array}$ & $\begin{array}{l}3.26 \mathrm{~b} ; 3.68 ; \\
4.35\end{array}$ & $1.00(0.36)$ & $1.03(0.31)$ & $1.04(0.18)$ & $1.50(0.37)^{* *}$ & $0.77(0.17)^{\#}$ & $0.97(0.30)$ \\
\hline $\mathrm{PC}$ & Phosphocholine & $\begin{array}{l}3.22 \mathrm{~s} ; 3.62 \\
4.18\end{array}$ & $1.00(0.76)$ & $0.98(0.36)$ & $1.47(0.72)$ & $0.79(0.46)$ & $1.23(0.55)$ & $1.42(0.43)^{\$}$ \\
\hline PUF & $\begin{array}{l}\text { Polyunsaturated } \\
\text { FA }\end{array}$ & $2.82 b ; 5.33$ & $1.00(0.47)$ & $0.93(0.57)$ & $1.24(0.62)$ & $2.17(0.95)^{* *}$ & $0.64(0.32)^{\#}$ & $0.71(0.37)$ \\
\hline GSx & $\begin{array}{l}\text { Total glutathione } \\
\text { (reduced + oxi- } \\
\text { dized) }\end{array}$ & $\begin{array}{c}2.55 \mathrm{~m} ; 2.12 \\
2.99 ; 3.77\end{array}$ & $1.00(0.42)$ & $1.03(0.53)$ & $1.33(0.56)$ & $1.18(0.46)$ & $0.79(0.24)$ & $1.16(0.57)$ \\
\hline Gln & Glutamine & $\begin{array}{l}2.44 \mathrm{~m} ; 2.14 \\
\quad 3.77\end{array}$ & $1.00(0.46)$ & $2.13(1.74)$ & $1.76(1.30)$ & $0.68(0.46)$ & $1.31(0.88)$ & $1.19(1.02)$ \\
\hline Pyr & Pyruvate & $2.37 \mathrm{~s}$ & $1.00(0.65)$ & $0.95(0.56)$ & $1.47(0.67)$ & $0.89(0.42)$ & $1.03(0.52)$ & $1.16(0.78)$ \\
\hline Glu & Glutamate & $\begin{array}{c}2.35 \mathrm{~m} ; 2.04 \\
2.11 ; 3.77\end{array}$ & $1.00(0.77)$ & $1.05(0.52)$ & $1.23(0.76)$ & $0.84(0.29)$ & $0.97(0.16)$ & $1.07(0.58)$ \\
\hline Ala & Alanine & $1.46 \mathrm{~d} ; 3.77$ & $1.00(0.42)$ & $1.00(0.28)$ & $1.07(0.38)$ & $0.60(0.27)^{*}$ & $1.19(0.37)$ & $1.16(0.31)$ \\
\hline Lac & Lactate & $1.33 \mathrm{~d} ; 4.12$ & $1.00(0.53)$ & $1.30(0.49)$ & $1.20(0.29)$ & $1.15(0.74)$ & $1.03(0.32)$ & $1.66(0.50)^{\$ \$}$ \\
\hline $\mathrm{tFA}$ & $\begin{array}{l}\text { Total FA (satu- } \\
\text { rated + unsatu- } \\
\text { rated) }\end{array}$ & $\begin{array}{r}1.29 \mathrm{~b} ; 1.59 \\
2.03 ; 2.25\end{array}$ & $1.00(0.32)$ & $0.81(0.28)$ & $0.91(0.53)$ & $1.77(0.83)^{*}$ & $0.64(0.37)^{\#}$ & $0.90(0.42)$ \\
\hline Prp & Propionate & $1.05 \mathrm{t} ; 2.17$ & $1.00(0.61)$ & $0.84(0.33)$ & $1.03(0.50)$ & $0.59(0.29)^{*}$ & $1.24(0.41)$ & $1.16(0.23)$ \\
\hline
\end{tabular}

First and second columns, metabolites; third column, chemical shift of metabolite NMR signals. The signal used for metabolite quantification is given with its coupling pattern specified using a letter: s, singlet; d, doublet; t, triplet; m, multiplet; b, broad signal. Fourth to seventh columns, concentration relative to NL (fold change vs. NL); eighth and ninth columns, concentration relative to LHON (fold change vs. LHON); L-IDE or L-RSV concentration relative to $\mathrm{NL}=(\mathrm{L}-\mathrm{IDE}$ or $\mathrm{L}-\mathrm{RSV}$ concentration relative to $\mathrm{LHON}) \times(\mathrm{LHON}$ concentration relative to NL)

$* \mathrm{p}<0.05$ LHON vs. NL; **p $<0.01$ LHON vs. NL

$\$_{\mathrm{p}}<0.05$ L-RSV vs. LHON; ${ }^{\$} \mathrm{p}<0.01 \mathrm{~N}-\mathrm{RSV}$ vs. NL or L-RSV vs. LHON

${ }^{\#} \mathrm{p}<0.05, \mathrm{~L}-\mathrm{IDE}$ vs. LHON (Student's $t$ test)

It is a potent antioxidant and prevents ROS-induced mitochondrial dysfunction. From the above discussion on FA in LHON, the response to IDE probably testifies the improvement of mitochondrial betaoxidation and the reduction of LD formation. In addition, IDE induced no significant effects on AA levels, thus should not target AA transport or mitophagy.
The other treatment, RSV, increased CI activity in LHON, in agreement with other reports in CID (Lopes Costa et al. 2014). FA signals were partially decreased in LHON treated by RSV, in favor of an improvement in mitochondrial/peroxisomal beta-oxidation and/or reduction of LD. This is agreement with reports on RSV (Massimi et al. 2012). RSV increased AA levels. From our discussion about AA levels in LHON, this supports that RSV improves cytosolic AA 

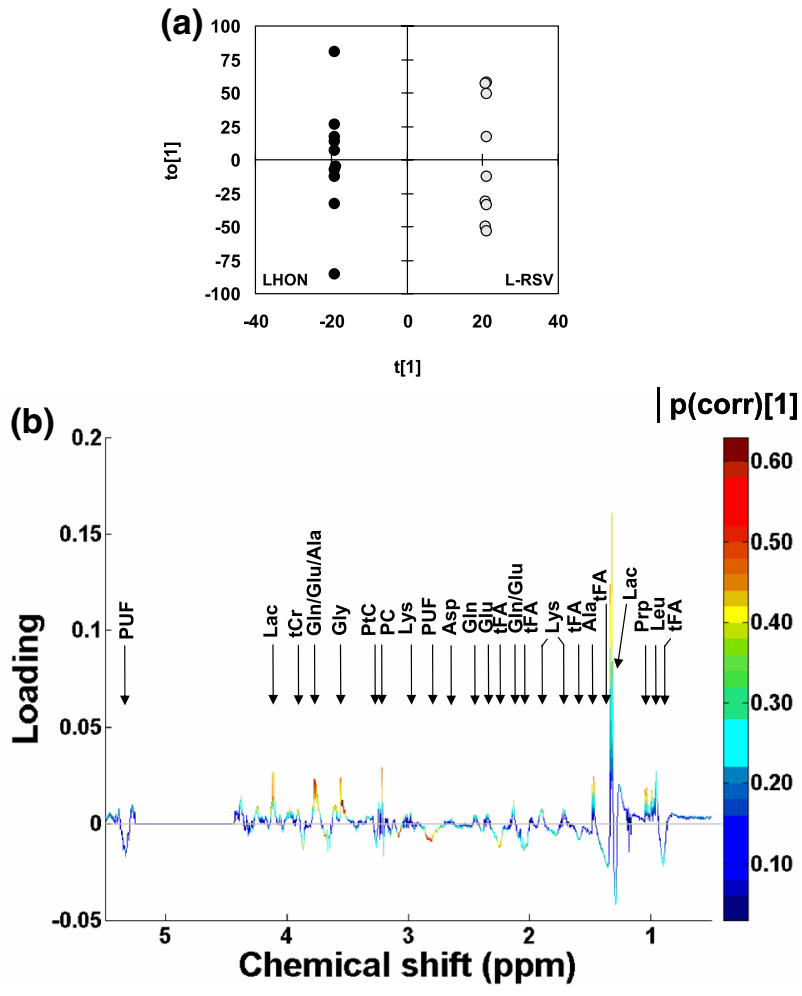

Fig. 3 a, b OPLS-DA of $1 \mathrm{D}{ }^{1} \mathrm{H}-\mathrm{NMR}$ spectra of L-RSV vs. LHON. $\mathrm{LHON}, \mathrm{n}=10$, vs. $\mathrm{L}-\mathrm{RSV}, \mathrm{n}=9 . \mathrm{R} 2 \mathrm{X}=0.96, \mathrm{R} 2 \mathrm{Y}=1$ and $\mathrm{Q} 2=0.54$, 1 predictive and 7 orthogonal components. a Scores plot (t[1] vs. t[0]). Black dots, LHON; light grey dots, L-RSV. b Loadings plot on the predictive component. Main assignment is given. Full assignment and metabolite abbreviations, see Table 1; positive loading, increased in L-RSV; negative loading, decreased in L-RSV; color-coding, strength of the correlation coefficient

transport and mitophagy. Indeed several reports showed that RSV increases Glu and AA transporter activity in astrocytes and glioma cells (Bellaver et al. 2016), and activates autophagy (Wu et al. 2011).

Intriguingly, RSV increased markedly Lac level in LHON. This could be due to downregulation of monocarboxylate transporters. However, the beneficial impact of RSV on cellular bioenergetics is quite complex, resulting from changes in redox homeostasis, activation of AMPK, stimulation of mitogenesis and mitophagy, but also from mitochondrial uncoupling and inhibition of ATP synthase/ Complex V (de Oliveira et al. 2016). A hypothesis is that RSV could make Complex V to function in reverse as an ATPase (Distelmaier et al. 2015), thus consume ATP of glycolytic origin, increase Lac production and intracellular Lac level. ATPases are also found in lysosome membranes. They may be activated by RSV which increases lysosome fusion with autophagosomes, thus contributing to increase cytosolic AA levels.

\subsection{Implications for LHON}

The main target of LHON is RGC. Although extrapolation from skin fibroblasts to RGC needs to be cautious, findings of this study support hypotheses on RGC sensitivity to cell death in LHON. Bioenergetics alteration does not satisfactorily explain loss of RGC (Kirches 2011), in agreement with preservation of ATP levels in LHON in our study. Alternatively, reduction of cytosolic AA levels that may originate from decreased plasma membrane uptake could contribute to increase extracellular Glu levels that cause excitotoxicity. Also the increase of LD, autophagosomes, and mitochondria-ER interactions could impair mitochondrial trafficking to the sites of energetic needs, another cause of RGC loss. Importantly, metabolic response to mitochondria-targeted treatments was consistent with (IDE) or in favor of (RSV) the beneficial effect of these agents in LHON.

\section{Overall conclusions}

The present NMR spectroscopy-based metabolomics study shows that LHON fibroblasts exhibit metabolic alterations including intracellular lipid increase and AA decrease. These abnormalities were corrected partly by mitochondria-targeted treatments, and could be explained by adaptive changes to CID, including inhibition of betaoxidation, formation of $\mathrm{LD}$, decreased cytosolic AA transport and activation of autophagy. CID-associated pathways in LHON fibroblasts can help to explain CID expression in LHON, in response to CI-targeted drugs or in diseases including neurodegeneration and cancer.

Acknowledgements The authors wish to thank Dr. Dominique Loiseau, INSERM U771, 49000 Angers, France, for providing LHON fibroblasts.

Author contributions The authors have equally contributed to this work.

\section{Compliance with ethical standards}

Conflict of interest The authors declare that they have no conflict of interest.

Ethical approval All procedures performed in studies involving human participants were in accordance with the 1964 Helsinki declaration and its later amendments and approved by the Ethics Committee of the University Hospital of Angers.

Informed consent Informed consent was obtained from all individual participants included in the study.

Open Access This article is distributed under the terms of the Creative Commons Attribution 4.0 International License (http://creat ivecommons.org/licenses/by/4.0/), which permits unrestricted use, 
distribution, and reproduction in any medium, provided you give appropriate credit to the original author(s) and the source, provide a link to the Creative Commons license, and indicate if changes were made.

\section{References}

Angebault, C., Gueguen, N., Desquiret-Dumas, V., Chevrollier, A., Guillet, V., Verny, C., et al. (2011). Idebenone increases mitochondrial complex I activity in fibroblasts from LHON patients while producing contradictory effects on respiration. $B M C$ Research Notes, 4, 557. https://doi.org/10.1186/1756-0500-4-557.

Bayet-Robert, M., Loiseau, D., Rio, P., Demidem, A., Barthomeuf, C., Stepien, G., \& Morvan, D. (2010). Quantitative two-dimensional HRMAS 1H-NMR spectroscopy-based metabolite profiling of human cancer cell lines and response to chemotherapy. Magnetic Resonance in Medicine, 63(5), 1172-1183. https://doi. org/10.1002/mrm.22303.

Baykal, A. T., Jain, M. R., \& Li, H. (2008). Aberrant regulation of choline metabolism by mitochondrial electron transport system inhibition in neuroblastoma cells. Metabolomics, 4(4), 347-356. https://doi.org/10.1007/s11306-008-0125-3.

Bellaver, B., Bobermin, L. D., Souza, D. G., Rodrigues, M. D. N., de Assis, A. M., Wajner, M., et al. (2016). Signaling mechanisms underlying the glioprotective effects of resveratrol against mitochondrial dysfunction. Biochimica et Biophysica Acta (BBA) Molecular Basis of Disease, 1862(9), 1827-1838. https://doi. org/10.1016/j.bbadis.2016.06.018.

Beretta, S., Mattavelli, L., Sala, G., Tremolizzo, L., Schapira, A. H. V., Martinuzzi, A., et al. (2004). Leber hereditary optic neuropathy mtDNA mutations disrupt glutamate transport in cybrid cell lines. Brain: A Journal of Neurology, 127(Pt 10), 2183-2192. https:// doi.org/10.1093/brain/awh258.

Boren, J., \& Brindle, K. M. (2012). Apoptosis-induced mitochondrial dysfunction causes cytoplasmic lipid droplet formation. Cell Death and Differentiation, 19(9), 1561-1570. https://doi. org/10.1038/cdd.2012.34.

Chao de la Barca, J. M., Simard, G., Amati-Bonneau, P., Safiedeen, Z., Prunier-Mirebeau, D., Chupin, S., et al. (2016). The metabolomic signature of Leber's hereditary optic neuropathy reveals endoplasmic reticulum stress. Brain, 139(11), 2864-2876. https://doi. org/10.1093/brain/aww222.

de Oliveira, M. R., Nabavi, S. F., Manayi, A., Daglia, M., Hajheydari, Z., \& Nabavi, S. M. (2016). Resveratrol and the mitochondria: From triggering the intrinsic apoptotic pathway to inducing mitochondrial biogenesis, a mechanistic view. Biochimica et Biophysica Acta, 1860(4), 727-745. https://doi.org/10.1016/j.bbagen.2016.01.017.

Distelmaier, F., Valsecchi, F., Liemburg-Apers, D. C., Lebiedzinska, M., Rodenburg, R. J., Heil, S., et al. (2015). Mitochondrial dysfunction in primary human fibroblasts triggers an adaptive cell survival program that requires AMPK- $\alpha$. Biochimica et Biophysica Acta (BBA) - Molecular Basis of Disease, 1852(3), 529-540. https://doi.org/10.1016/j.bbadis.2014.12.012.

Dombi, E., Diot, A., Morten, K., Carver, J., Lodge, T., Fratter, C., et al. (2016). The m.13051G $>$ A mitochondrial DNA mutation results in variable neurology and activated mitophagy. Neurology, 86(20), 1921-1923. https://doi.org/10.1212/WNL.0000000000002688.

Graham, S. F., Kumar, P. K., Bjorndahl, T., Han, B., Yilmaz, A., Sherman, E., et al. (2016). Metabolic signatures of Huntington's disease (HD): (1)H NMR analysis of the polar metabolome in postmortem human brain. Biochimica et Biophysica Acta, 1862(9), 1675-1684. https://doi.org/10.1016/j.bbadis.2016.06.007.

He, Q., Wang, M., Petucci, C., Gardell, S. J., \& Han, X. (2013). Rotenone induces reductive stress and triacylglycerol deposition in
$\mathrm{C}_{2} \mathrm{C}_{12}$ cells. The International Journal of Biochemistry \& Cell Biology, 45(12), 2749-2755. https://doi.org/10.1016/j.bioce 1.2013.09.011.

Janzer, A., German, N. J., Gonzalez-Herrera, K. N., Asara, J. M., Haigis, M. C., \& Struhl, K. (2014). Metformin and phenformin deplete tricarboxylic acid cycle and glycolytic intermediates during cell transformation and NTPs in cancer stem cells. Proceedings of the National Academy of Sciences of the United States of America, 111(29), 10574-10579. https://doi.org/10.1073/ pnas. 1409844111.

Kirches, E. (2011). LHON: Mitochondrial mutations and more. Current Genomics, 12(1), 44-54. https://doi.org/10.2174/1389202117 94520150

Leong, D. W., Komen, J. C., Hewitt, C. A., Arnaud, E., McKenzie, M., Phipson, B., et al. (2012). Proteomic and metabolomic analyses of mitochondrial complex I-deficient mouse model generated by spontaneous B2 short interspersed nuclear element (SINE) insertion into NADH dehydrogenase (ubiquinone) $\mathrm{Fe}-\mathrm{S}$ protein 4 (Ndufs4) gene. The Journal of Biological Chemistry, 287(24), 20652-20663. https://doi.org/10.1074/jbc.M111.327601.

Loiseau, D., Chevrollier, A., Verny, C., Guillet, V., Gueguen, N., Pou de Crescenzo, M.-A., et al. (2007). Mitochondrial coupling defect in Charcot-Marie-Tooth type 2A disease. Annals of Neurology, 61(4), 315-323. https://doi.org/10.1002/ana.21086.

Lopes Costa, A., Le Bachelier, C., Mathieu, L., Rotig, A., Boneh, A., De Lonlay, P., et al. (2014). Beneficial effects of resveratrol on respiratory chain defects in patients' fibroblasts involve estrogen receptor and estrogen-related receptor alpha signaling. Human Molecular Genetics, 23(8), 2106-2119. https://doi.org/10.1093/hmg/ddt603.

Massimi, M., Tomassini, A., Sciubba, F., Sobolev, A. P., Devirgiliis, L. C., \& Miccheli, A. (2012). Effects of resveratrol on HepG2 cells as revealed by $1 \mathrm{H}-\mathrm{NMR}$ based metabolic profiling. Biochimica et Biophysica Acta (BBA) - General Subjects, 1820(1), 1-8. https:// doi.org/10.1016/j.bbagen.2011.10.005.

Mathieu, L., Costa, A. L., Le Bachelier, C., Slama, A., Lebre, A.-S., Taylor, R. W., et al. (2016). Resveratrol attenuates oxidative stress in mitochondrial Complex I deficiency: Involvement of SIRT3. Free Radical Biology \& Medicine, 96, 190-198. https://doi. org/10.1016/j.freeradbiomed.2016.04.027.

Mimaki, M., Wang, X., McKenzie, M., Thorburn, D. R., \& Ryan, M. T. (2012). Understanding mitochondrial complex I assembly in health and disease. Biochimica et Biophysica Acta, 1817(6), 851862. https://doi.org/10.1016/j.bbabio.2011.08.010.

Morgan, P. G., Higdon, R., Kolker, N., Bauman, A. T., Ilkayeva, O., Newgard, C. B., et al. (2015). Comparison of proteomic and metabolomic profiles of mutants of the mitochondrial respiratory chain in Caenorhabditis elegans. Mitochondrion, 20, 95-102. https://doi.org/10.1016/j.mito.2014.12.004.

Worth, A. J., Basu, S. S., Snyder, N. W., Mesaros, C., \& Blair, I. A. (2014). Inhibition of neuronal cell mitochondrial complex I with rotenone increases lipid $\beta$-oxidation, supporting acetyl-coenzyme A levels. The Journal of Biological Chemistry, 289(39), 2689526903. https://doi.org/10.1074/jbc.M114.591354.

Wu, Y., Li, X., Zhu, J. X., Xie, W., Le, W., Fan, Z., et al. (2011). Resveratrol-activated AMPK/SIRT1/autophagy in cellular models of Parkinson's disease. Neuro-Signals, 19(3), 163-174. https://doi. org/10.1159/000328516.

Xu, Q., Vu, H., Liu, L., Wang, T.-C., \& Schaefer, W. H. (2011). Metabolic profiles show specific mitochondrial toxicities in vitro in myotube cells. Journal of Biomolecular NMR, 49(3-4), 207-219. https://doi.org/10.1007/s10858-011-9482-8.

Zhang, L.-N., Hao, L., Wang, H.-Y., Su, H.-N., Sun, Y.-J., Yang, X.-Y., et al. (2015). Neuroprotective effect of resveratrol against glutamate-induced excitotoxicity. Advances in Clinical and Experimental Medicine: Official Organ Wroclaw Medical University, 24(1), 161-165. 